\title{
Elementos para un debate contemporáneo sobre la educación abierta
}

\author{
HÉCTOR BARRÓN SOTO \\ (Coordinación de Universidad Abierta y Educación a Distancia \\ Universidad Nacional Autónoma de México)
}

RESUMEN: Se argumenta en torno de las discusiones tradicionales sobre la educación abierta, sobre su origen, desarrollo y tendencias. A partir de la identificación de estos elementos se plantea la necesidad de reubicar estas discusiones en un nuevo contexto, que haga más comprensible la misión de este tipo de educación y, sobre todo, cómo instrumentar su viabilidad y cuáles serían las condiciones para operarla.

Se propone que el debate sobre la educación abierta vuelva a recuperar aspectos más elementales, de forma que el de la educación abierta sea un problema centrado casi exclusivamente en aspectos técnicos, fundamentalmente en el intento de dar solución a la pregunta: ¿cómo debe organizarse la educación abierta para que sea posible la interpretación y la recreación del conocimiento? A este respecto se proponen dos principios para el ejercicio de la educación abierta: la libertad y la democracia, y se plantea una aportación en el escenario de la educación iberoamericana: la libertad de aprendizaje.

Educación abierta - filosofía educativa - educación abierta en Iberoamérica - práctica educativa libertad de aprendizaje - teorías de educación abierta.

ABSTRACT: This article deals with the traditional discussions around distance education, its origins, development and trends. Beginning with the identification of these elements, it is argued that these discussions must be placed in a new context that makes easier to understand the mission of this kind of education and, especially, how to improve its viability and the conditions to make it operative.

It is proposed that the debate around distance education return to more elemental issues, so that the discussion around open education should deal with technical aspects almost exclusively, in particular those that try to give answer to the question: How should open education be organized so that it is possible to reinterpretate and recreate knowledge? Two principles are proposed in order to improve distance education: freedom and democracy; and a scenario for iberoamerican education is proposed: learning freedom.

Open education - Educational Philosophy - Open education in Iberoamerica - Educational practice - learning freedom - Theories of open education 


\section{UNA BREVE HISTORIA DE LA IDEA DE LA EDUCACIÓN ABIERTA}

Una de las preguntas iniciales con que se construyen las indagatorias sobre la educación abierta es la que se refiere a su origen y a su naturaleza: ¿qué es la educación abierta?

Un gran número de autores señala que el origen de la educación abierta se encuentra en el aumento entre la demanda por acceder a instituciones tradicionales de educación y la poca oferta para dar cumplimiento a esta demanda, sobre todo en el periodo posterior a la Segunda Guerra Mundial; otro factor que mencionan es el de la necesidad de preparar cuadros especializados que ya estaban trabajando, de donde había que considerar alumnos que tuvieran la posibilidad de trabajar y estudiar al mismo tiempo. Este tipo de análisis coloca al contexto socioeconómico como el generador de la educación abierta institucional. Otros autores destacan la influencia de la tecnología para la expansión de este tipo de instituciones.

Otros más resaltan factores de tipo ideológico, de donde se trata de dar expresión a principios como el de la «democratización de la enseñanza» y la «justicia social frente a las desigualdades educativas». De esta manera se preservaba la idea de que a través de la obtención de ciertos niveles de conocimiento, a través de la obtención de títulos universitarios, podía accederse casi de manera inmediata y natural a niveles sociales más estables.

Cualquiera de estos enfoques enriquece el análisis del surgimiento institucional de este tipo de educación. Sin embargo, ninguno de ellos, por sí solo, llega a dar satisfacción a la pregunta inicial acerca de qué es la educación abierta, aunque todos coinciden en que el momento histórico para que se conjugaran las condiciones que la hicieran posible está referido particularmente a la década de los años cincuenta.

Existe otro análisis que se ha venido fortaleciendo en los últimos años, y que toma como referentes sobre todo dos fenómenos: primero, las nuevas formas de organización del conocimiento y, segundo, las diversas maneras y posibilidades de presentación del conocimiento por medios tecnológicos. Este análisis se ha desprendido de la necesidad por comprender el entorno en que se propone la iniciativa mundial ${ }^{1}$ para conformar lo que se ha dado en llamar la sociedad de la información.

\footnotetext{
A partir de los planteamientos vertidos en el «Libro Blanco sobre crecimiento, competitividad y empleo: retos y oportunidades para ingresar al siglo xxi, elaborado en Bruselas en diciembre de 1993, y del Informe Bangemann («Europa y la sociedad global de la información. Recomendaciones del Grupo Bangemann al Consejo Europeo, mayo de 1994), se expresaron varias iniciativas regionales y de comunidades, con el propósito de inducir las consecuencias concretas del programa de la sociedad de la información. El carácter internacional de estas iniciativas se dio con la reunión de los países miembros del G7, en Bruselas, destinado precisamente a idear una estrategia mundial para avanzar hacia esta sociedad de la información.
} 
El primer fenómeno se sostiene sobre las derivaciones concretas de la interdisciplinariedad, que propició por lo menos desde 1946 la constitución formal de las ciencias cognitivas, de donde a través de la conjugación de diversas disciplinas como matemáticas, fisiología, anatomía, psicología, antropología, lingüística y filosofía, se planteó el análisis de problemas en conjunto. Este fenómeno obligó a considerar que los problemas del conocimiento que antes eran abordados casi exclusivamente por la filosofía, pudieran ser también analizados por las ciencias. Lo que antes pertenecía a la esfera de estudio de la lógica y la ética, pasó a pertenecer también a la de las matemáticas; en suma, el conocimiento mismo pasó a ser entonces también un objeto de estudio, lo que ha conducido a una cada vez más extendida revolución de la información, de la comunicación, la selección, la decisión y la acción.

Uno de los precursores internacionales de la teoría de la interdisciplinariedad, el físico de origen húngaro John Von Neumann, intentó construir un sistema de conocimientos más próximo a lo objetivo, inspirándose en los métodos de la teoría de las probabilidades, de la termodinámica, de la teoría de la información y del análisis matemático.

Para dar una idea precisa de este fenómeno, baste recordar que en el mundo de hoy cada cinco años se duplica el total de la información existente.

El segundo fenómeno se deriva del rápido desarrollo de las tecnologías, y sobre todo del de la telemática, en este sentido entendida como el uso combinado de las telecomunicaciones, la televisión y la informática, que está ya incidiendo en la modulación de nuevas formas de comunicación e interrelación social más allá de las limitaciones geográficas y temporales, así como en nuevas y diversas formas de organización del conocimiento, causando distintos impactos.

Esta convergencia cada día más estrecha entre nuevas formas de organización del conocimiento y nuevos formatos de presentación, ha acelerado también el que la información tenga un incremento exponencial, que tenga una durabilidad más breve, que se organice en formas más complejas, que permita múltiples interpretaciones, que facilite su disponibilidad, y que permita una aplicación más inmediata.

De aquí que la expresión del conocimiento para la resolución de problemas haya incrementado de manera importante el valor del mismo conocimiento en el proceso de producción, de ahí que núcleos importantes de la sociedad demanden de manera sostenida la ampliación en el acceso a la educación profesional, por un lado, y la adecuación del modelo de servicio profesional al campo real de aplicación de los conocimientos en el trabajo, por el otro.

Las universidades e instituciones de cultura que antes habían llevado a cabo su labor de adquisición y transmisión de conocimientos de una manera armónica, equilibrando su función de transmisión de información con la del desarrollo 
de habilidades para la toma de decisiones profesionales, enfrentaron entonces un proceso de acumulación de actividades informativas, en detrimento de las actividades de índole formativa o de procuración de la ética que la institución deseaba fomentar en sus alumnos y en sus profesores.

Este proceso ha ido acentuándose de manera paulatina, aunque las instituciones de educación han enriquecido sus formas de trabajo con innovaciones tales como el trabajo en los laboratorios y los seminarios, pero el problema fundamental sigue siendo el siguiente: ¿cómo enfrentar un proceso de acelerado incremento en el volumen en la información y de generación de nuevas disciplinas con una estructura educativa poco flexible a los cambios?

La suma de estos aspectos derivó claramente en la percepción social de que algo estaba fallando en las instituciones de educación y el sistema social de contratación de profesionistas. Los egresados profesionales, al incorporarse a los centros de trabajo, debían dedicar un periodo cada vez más largo en la adquisición de la experiencia necesaria para cumplir con las exigencias peculiares del trabajo. Más adelante, estos mismos trabajadores profesionales advertirían que, a diferencia de sus padres o abuelos, debían además mantenerse actualizados en cuanto a la generación de nuevos conocimientos de su disciplina, y en cuanto a las variaciones en su aplicación para la toma de decisiones.

En este contexto es que periódicamente se afirma que la educación, sus componentes y formas de vinculación están en crisis, tal como están en crisis ideologías, valores, paradigmas y formas de interpretación de la realidad. En este contexto también es que se procura la reflexión sobre las condiciones que llevan a un modelo educativo a su agotamiento, y las estrategias para abrir este modelo educativo, es decir, para asegurarle una transformación que le permita mantener las posibilidades de superar sus vicios de estructura y de organización.

Por lo pronto, en vista de que nadie puede esperar una respuesta a la pregunta ¿qué es la educación abierta?, para superar los problemas de los modelos de educación exclusivamente presenciales, y en vista también de que parámetros como los de eficiencia y competitividad repercuten para cultivar la idea de que quien posee el conocimiento no es el profesionista más calificado sino aquél que sabe utilizar la información en la resolución de problemas prácticos, se analiza a la educación abierta desde otras perspectivas. Una de ellas es la histórica.

El surgimiento y la conformación de los sistemas de educación abierta no ha sido un fenómeno exclusivo de un país o de un área geográfica determinada, ni siquiera de una época histórica definida. Más bien ha sido una práctica de renovación de los principios clásicos de la enseñanza y del aprendizaje que bajo determinadas condiciones se fortalece y se transforma. Su aparición, por ello, es producto del agotamiento de lo que por antonomasia estamos llamando práctica docente cerrada, aún cuando en la práctica no pueda existir una práctica docente totalmente cerrada. 
La educación ha conocido diversos momentos de apertura que, en sí, no han consolidado sino adecuaciones a etapas históricas particulares, al término de los cuales esa apertura adquiere una propiedad de oclusión. Asimismo, la suma de estos momentos de apertura tampoco significa una totalidad de aperturas.

Particularmente en América Latina hubo intentos muy sentidos —en especial por parte de uno de los sujetos de la práctica docente: los estudiantes-y más o menos sistematizados acerca de la necesidad de transformar la práctica educativa desde principios de siglo. En un recuento que José Carlos Mariátegui realiza acerca de la Reforma Universitaria dice:

El congreso internacional de estudiantes de México de 1921 propugnó: $1^{\circ}$. La participación de los estudiantes en el gobierno de las universidades; $2^{\circ}$. La implantación de la docencia libre y la asistencia libre. Los estudiantes de Chile declararon su adhesión a los siguientes principios: $1^{\circ}$. Autonomía de la Universidad, entendida como institución de los alumnos, profesores y diplomados; $2^{\circ}$. Reforma del sistema docente, mediante el establecimiento de la docencia libre y, por consiguiente, de la asistencia libre de los alumnos a las cátedras, de suerte que en el caso de enseñar dos maestros una misma materia la preferencia del alumnado consagre libremente la excelencia del mejor; $3^{\circ}$. Revisión de los métodos y del contenido de los estudios, y $4^{\circ}$. Extensión universitaria, actuada como medio de vinculación efectiva de la Universidad con la vida social. Los estudiantes de Cuba concretaron en 1923 sus reivindicaciones en esta fórmula: a. Una verdadera democracia universitaria; b. Una verdadera renovación pedagógica y científica; c. Una verdadera popularización de la enseñanza. Los estudiantes de Colombia reclamaron, en su programa de 1924, la organización de la Universidad sobre bases de independencia, de participación de los estudiantes en su gobierno, y de nuevos métodos de trabajo. 'Que al lado de la cátedra — dice el programa- funcione el seminario, se abran cursos especiales, se creen revistas. Que al lado del maestro titular haya profesores agregados y que la carrera del magisterio exista sobre bases que aseguren su porvenir y den acceso a cuantos sean dignos de tener una silla en la Universidad'.

Ciertos logros de esta práctica educativa, cuando fue posible que fuera realmente una práctica, como la ampliación de los cuerpos colegiados de las instituciones hacia algunos sectores de la comunidad que la integran, y los derechos del individuo ante la institución, como la libertad de cátedra, tuvieron que ser incorporados al viejo esquema de la práctica educativa, favoreciéndose así un escenario menos de confrontación de prácticas educativas que de cooperación, aunque con la tendencia de estabilizar un periodo de transición que condujera finalmente hacia un tipo de práctica educativa realmente abierta.

Cada institución de educación superior de Iberoamérica interpretó los signos de esta práctica educativa en diversos niveles, y aplicó generalmente la idea de la institución como mentora de los grupos de aprendizaje, induciendo una vuelta hacia una práctica educativa oclusiva, aunque participativa. 
Fue en el año de 1968 que se agudizó la incapacidad de la práctica educativa oclusiva hasta el grado paradójico de incentivar movimientos en su contra. A diferencia de Estados Unidos, en Europa y en América Latina los movimientos estudiantiles estuvieron marcadamente politizados e inclinados hacia la izquierda, además de que se incluyó la autogestión como un punto político importante de la agenda de demandas hacia la institución y hacia el Estado.

El saldo positivo de esta oleada contra la práctica educativa tal cual estaba concebida, fue que se «modernizó» el problema de la educación, es decir, que los grupos de aprendizaje cobraron presencia y adquirieron voz dentro de la institución. Pero en todo lo demás la sustancia de la práctica educativa oclusiva no había desaparecido.

Quizá una de las singularidades de la práctica educativa oclusiva en Latinoamérica haya sido la permanencia: si bien la institución ha dado espacios de expresión a los grupos de aprendizaje, no ha renunciado a preservar su estatus de mentora, del mismo modo que el Estado no ha dejado de aspirar a mantener su función de benefactor social, ya sea a través del dominio unipartidista, de los constantes llamados a los golpes de estado, del autoritarismo revolucionario o de la resistencia a abandonar las reminiscencias fascistas.

Como quiera que sea, es improbable que las instituciones educativas deseen alejarse de su función como mentoras, pero hay circunstancias externas que las están obligando a realizar más de un cambio para sobrevivir.

Hay otros factores decisivos que refuerzan las tendencias institucionales para incorporar un mayor número de principios de la práctica educativa abierta a su viejo modelo: la explosión de la información y su presentación en nuevas estructuras de conocimiento, la demografía y su relación con los nuevos esquemas del trabajo especializado, la identidad cultural, el cuestionamiento a la nación, y la iniquidad económica y social, entre los más importantes.

Algunos de estos factores pueden ser analizados por medio de la interrelación entre la democracia y el liberalismo, entre la preservación de los grupos homogéneos en sí y la valoración de los individuos diferentes entre sí, entre el fomento a la atención de los grupos de aprendizaje y la necesidad de particularizar los requerimientos del individuo de aprendizaje. La intensidad y profundidad en que pueda lograrse una práctica educativa abierta dependerá, con mucho, de la dimensión que podamos darle a la diferencia, es decir, al individuo de aprendizaje dentro de un grupo de aprendizaje y, más aún, a este grupo de aprendizaje dentro de una comunidad de aprendizaje.

La práctica educativa abierta contemporánea encuentra sus raíces en dos vertientes de la cultura occidental: la libertad y la democracia. Para que los dos términos tengan plenitud de expresión, deben ajustarse a las condiciones im- 
puestas por su ejecución: la opcionalidad para seleccionar de entre múltiples recursos y condiciones dispuestos para propiciar el aprendizaje; el margen suficiente para disentir de los discursos, y las posibilidades de incluir nuevos miembros y nuevos recursos a los ambientes de aprendizaje, entre los más significativos, es decir, permitir la generación de diversas formas de estructuración del conocimiento para contener la acelerada generación de nuevos saberes.

\section{LA NATURALEZA CRÍTICA DE LA PRÁCTICA EDUCATIVA ABIERTA}

En una somera recapitulación acerca del estado actual de las interpretaciones sobre la naturaleza de la práctica educativa, prevalece un consenso acerca de la crisis que permea sus actividades, sin embargo, dentro de las principales discusiones contemporáneas para explicar las causas de esta crisis destaca una disconformidad que suele expresarse entre la concepción de la práctica educativa como una forma de alcanzar una visión del mundo y sistematizarla, y una práctica educativa como una técnica para reinterpretar la realidad y transformar el mundo; ambos grupos de argumentos son consecuencia de posturas aún más irreductibles que dejan traslucir un fondo interesante para entender el sentido de la educación superior en nuestros días, no solamente por lo que se refiere a la metodología, sino particularmente por lo que hace al conocimiento y a su organización.

Las premisas que soportan la identidad de ambos grupos argumentales pueden personalizar a uno como el de los conservadores y al otro como el de los inconformes. Dentro del primer grupo pueden encontrarse formulaciones donde se propone que para incluir un conocimiento al programa de estudios éste debe ser de alta calidad y trascender su momento histórico; que existen normas objetivas e intersubjetivas con base en las cuales es posible argumentar por qué un conocimiento es de alta calidad y en qué medida trasciende su momento histórico; que una de las intenciones de la práctica docente es la de cuestionar las contingencias culturales de los estudiantes para incorporarlos a una comunidad intelectual más amplia; que es posible armonizar las capacidades e intereses del individuo en la medida en que éste cobre conciencia de pertenecer a una comunidad participante de una cultura humana universal; que dentro de las funciones primigenias de la práctica docente está la crítica de sí y la crítica de los sujetos que en ella participan; que existen preceptos verdaderos a los que deben corresponder enunciados verdaderos.

Los argumentos opuestos a los anteriores promueven la idea de que los individuos que participan en la práctica docente, en posición de enseñantes o estudiantes, están definidos por su origen étnico, de clase y de sexo, y que esta definición es irrenunciable, por lo que deben preservar su identidad en relación con su pertenencia a una comunidad determinada; que todos los conocimien- 
tos tienen el mismo valor, e intelectualmente son iguales; que la representatividad debe ser el criterio tanto para seleccionar los conocimientos que se establecerán en los programas de estudios, como para la composición del cuerpo docente; que la misión original de toda práctica docente es política y tiende hacia el cambio social, por tanto concibe a la universidad como una vía para incorporar a los estudiantes a una democracia multicultural; que las normas y los patrones de calificación son imagen de las condiciones históricas locales, de donde no es posible que sean ni objetivos ni desinteresados ni universales; que la verdad es un evento en permanente construcción; que una de las misiones de la práctica docente debe ser la de la independencia de criterios con respecto a los de la institución.

En opinión del filósofo norteamericano John R. Searle, el grupo de los argumentos inconformistas tiene varias premisas insostenibles: la primera es que cuando utilizamos un lenguaje público para comunicarnos, damos por descontado que existe una realidad previa a la que se alude, por lo tanto, el realismo metafísico que atacan los inconformes está presente desde el mismo lenguaje que utilizan; la segunda es que el lenguaje también presupone normas lógicas de construcción y argumentación, por lo tanto resultaría ocioso cuestionar la existencia de normas objetivas de racionalidad; la tercera se desprende del hecho de que prácticamente cualquier actividad humana tiene derivaciones políticas, de donde la educación no es la excepción pero tampoco podría afirmarse que es su fin; por último, la representatividad es un criterio secundario dependiente de la alta calidad y sus normas, aunque como tal es un criterio deseable en la medida en que pretende relacionar las variedades de experiencias del ser humano.

Por la parte del grupo argumental de los conservadores, Searle no refuta en esencia sus presupuestos, lo que observa es que hay una inercia hacia el anquilosamiento y la decadencia que puede observarse cuando las universidades fomentan el clientelismo o favorecen el amiguismo y la consolidación de grupos homologados, o cuando la institución se fija una misión diferente a la oficial. Lo que Searle encuentra es, antes que un mayor cuestionamiento a las posiciones de los conservadores, una desconfianza hacia el ideal de una educación integral y una incertidumbre sobre la factibilidad de conocer el éxito o el fracaso en la formación de los estudiantes, en suma, una crisis de autodefinición que afecta a los sujetos de la práctica docente: el grupo de enseñantes y la comunidad de estudiantes.

La práctica educativa abierta en la educación superior participa y comparte varios de los principios argumentales sostenidos tanto por los conservadores como por los inconformes, pero en su naturaleza tiende a conciliarlos y transformarlos bajo la perspectiva de que redefinirá consecutivamente su organización y las formas de relación entre cada uno de sus elementos, de forma que sus objeti- 
vos educativos puedan ser alcanzados a partir de situaciones de inicio diversas, pero siempre bajo normas objetivas que procuren la excelencia en las funciones de los docentes, y en el desempeño de los estudiantes, así como que amplíen las vías para que esos mismo docentes y estudiantes conozcan y recuperen sus experiencias de aprendizaje en un proceso continuo de concientización.

Bajo la teoría de los sistemas, la característica de la práctica educativa abierta que permite alcanzar un mismo estado final desde condiciones de inicio distintas, permite asimismo transitar hacia estadíos mejorados de organización que disminuyen la propensión de crecimiento de la entropía.

Para que ello sea posible es necesario identificar y consolidar diferentes mecanismos, que no necesariamente tienen que ser siempre los mismos, mediante los cuales sea posible establecer parámetros de autoadaptación a las condiciones variables de desarrollo; estos parámetros pueden ser, además de científicos, éticos y morales, y pueden encontrar expresión en amplios y flexibles sistemas de evaluación.

La descripción actual del fenómeno de la apertura en la educación está correlacionada con una dinámica de ubicación del conocimiento en la realidad metafísica a que alude John R. Searle, y que identifica a los sujetos de la práctica docente abierta, docentes y estudiantes, como individuos que se transforman al interpretar un modelo de la realidad y al decidir una recreación del mundo.

La interpretación y la decisión, la experiencia y la práctica, así, se recuperan en la práctica educativa abierta concibiendo la atención de grupos de aprendizaje integrados por estudiantes heterogéneos en su composición étnica y social, y con el apoyo de múltiples recursos e instrumentos metodológicos, contiguos o a distancia.

En este entorno crítico la práctica educativa abierta en la educación superior encuentra su sentido original en la combinación y conciliación entre el servicio que debe prestar a la sociedad democrática y a la satisfacción de las expectativas individuales: para servir a la democracia debe ser elitista, para cumplir las expectativas individuales debe ser diversa.

Por un lado, si se pretende alcanzar una alta calidad académica se debe seleccionar a los docentes mejor preparados y a los estudiantes más aptos para trabajar más arduamente. Por otro lado, si se desea ser dinámica debe integrar los conocimientos desde una amplia gama de fuentes y organizarlos y presentarlos en múltiples recursos, ya que alguno o algunos de ellos podrán adicionarse a la mejoría del trabajo de alguno o algunos de los estudiantes; la diversidad en sí como un principio no es sustentable sino como una vía real para acrecentar las posibilidades de acceder al conocimiento, y en tanto que sea posible es conveniente hacerlo. 


\section{LOS RIESGOS Y LAS DESVIACIONES DE LA PRÁCTICA EDUCATIVA ABIERTA}

La educación abierta parecería mantenerse desde una postura hermenéutica de la educación, sin pasar jamás a ser una práctica explícitamente desarrollada en tal o cual lugar, sin ser nunca un hecho ensayado y consagrado por el tiempo. No obstante, aún cuando así sea, puede aspirarse a instrumentarse como una práctica taciturna.

Es muy probable que cada paso que se de para el acercamiento con esta imagen de la práctica educativa sea iniciado por la Institución, de donde emanan el orden y la sistematización del conocimiento que se desea extender.

Así, la libertad y la democracia deben darse dentro de las instituciones, en nuestro caso, en las universidades. Muy probablemente en algún momento sea trazada una línea, símil quizá del Código Napoleónico, más allá de la cual no debería intervenir el poder institucional en la vida privada de los individuos, pues, como podría obviarse mencionarlo, la democracia es un ejercicio exclusivo del grupo, mientras la libertad pertenece a la esfera del individuo².

Sobre la base de un contrato del conocimiento que el alumno deberá estar en condición de manejar, y que el conjunto de materiales didácticos y recursos de apoyo para el aprendizaje deberá estar en condiciones de reforzar, el acto de la práctica educativa se construirá dentro de tales límites: esta geografía del conocimiento, sin embargo, no fatiga las formas para lograrlo: el alumno buscará o innovará las propias, las más ajustadas a su interés y capacidad, con la asistencia o sin ella, aún pese a los esfuerzos del docente, e incluso aún en contra de los intereses del docente. Este último deberá estar en capacidad de afianzar la tradición discursiva y de enlazarla hacia la originalidad del contexto.

Cada una de estas épocas ha privilegiado el entendimiento de la práctica educativa como un fenómeno de reproducción de las estructuras que representan la visión del mundo correspondiente: de una práctica educativa ocupada en afirmar la preeminencia del conocimiento como un corpus integrado en sí mismo, bastado en sus propios límites y como tal transmitido sin abrir un proceso de intercambio con otros sistemas de conocimiento exógenos, se pasó a la concepción de una práctica educativa fundada en la selección, organización y transmisión del

\footnotetext{
${ }^{2}$ Lo que quizá valdría la pena no obviar serían las consecuencias de un Estado benefactor ya no digamos concebido como el big brother orwelliano, sino para nosotros el utópico sistema sueco de principios de la década de los sesenta, donde el trabajador social disponía de los recursos legales para vigilar la pauta de consumo, los hábitos sexuales y el ritmo de vida del ciudadano que solicitaba beneficios, para plantear al Estado las proporciones concretas de beneficio que debieran otorgarse. En un arbitrario símil con las instituciones educativas, no tendríamos dificultad en imaginar las consecuencias de no definir el alcance de la esfera del grupo y la del individuo.
} 
Elementos para un debate contemporáneo

saber propio en concordancia con el reconocimiento de otras fórmulas de desarrollo de los sistemas de conocimiento; a su vez, de esta concepción transitamos a la del reconocimiento del saber ajeno como parte del propio sistema de conocimiento, de tal manera que su subsistencia está determinada por la apertura hacia sí mismo y hacia otros sistemas, y por el paulatino reconocimiento de que la sola transmisión del conocimiento no es en sí el fin de esta práctica educativa, ya que se han percibido los efectos inmediatos de un fenómeno de dimensiones todavía incalculables: el crecimiento exponencial del conocimiento ha iniciado un boom que está provocando que en estos momentos se duplique todo el saber científico y tecnológico que el hombre había acumulado desde su aparición hasta 1980.

Sin embargo, antes de seguir adelante habría que prevenir sobre las tendencias cerradas o totalitarias de los ambientes participativos, de consenso o democráticos.

A pesar de haber sido advertido desde tiempo atrás por autores como Jacob Talmon, y razonablemente explicado por Michel Foucault, ha sido hasta muy reciente que se ha tomado conciencia de las inclinaciones totalitarias del Estado, de donde no sólo la sociedad puede ser intolerante en un entorno democrático, sino que también puede serlo la educación en un ambiente participativo.

La sociedad como una presencia que ejercita su poder opresor sobre el individuo se ha dado en diversas ocasiones en el seno de las democracias florecientes, los ejemplos clásicos lo representan la turba anitidreyfusista en la república francesa y la cacería de brujas MacCarthiana. Todo esto se vincula con el proceso educativo porque, como dijimos, la práctica educativa se sostiene sobre la democracia y sobre la libertad: dos términos que únicamente pueden alcanzar la plenitud en el equilibrio de uno frente al otro.

En esta práctica educativa advertimos cómo la institución o el grupo de aprendizaje puede descubrir sus tendencias de dominación sobre los intereses de aprendizaje del individuo, alterando sus expectativas, modificando sus aspiraciones y en no pocos casos obstruyendo sus participaciones, en abono de un camino directo hacia lo que podría denominarse una "práctica educativa correcta», en los términos en que se ajusta a los intereses de una institución correlacionada con los intereses de un Estado: quien fuera de estos límites practica y busca otros intereses, es, primero, culpado, después marginado y finalmente expulsado.

En principio puede hablarse de dos fenómenos que promueven las inclinaciones absolutistas o cerradas de la práctica educativa abierta. El primer fenómeno puede ser explicado bajo la visión de Hegel sobre el Estado, de donde se desprendería que la institución es la depositaria y custodia de la libertad del individuo de aprendizaje. Los grupos de aprendizaje interactúan entre sí confrontando sus prejuicios e inhibiciones, es decir, regulando su dinámica de trabajo en 
armonía con una moralidad colectiva o percepción pública. Hegel suponía que esta pesada moralidad colectiva era necesaria para la formación del individuo, pero agregaba que esta formación debía ser completada por la institución, y no por el grupo de aprendizaje. De esta manera, las actividades que estimulan la presencia pública del individuo y ejemplifican la dedicación de éste para el aprendizaje, son practicadas en la esfera de la institución.

El espíritu que anima a los grupos de aprendizaje es el de preservar los límites de transformación de la moralidad colectiva, mismo que puede expresarse en la tendencia hacia vigilar los desplazamientos desinhibidos del individuo de aprendizaje y, en su momento, castigarlos. Sin embargo, hay una diferencia sustantiva entre la concepción de la institución como custodia de la libertad del individuo y su expresión como mentora de los grupos de aprendizaje y como servidora de los mismos grupos de aprendizaje.

Vayamos más despacio: para Hegel la consecuencia última de la dependencia de la institución con respecto de los grupos de aprendizaje sería precisamente el que la institución quedaría cautiva de los intereses de los grupos de aprendizaje y así sería incapaz de cumplir con su función de mentora; pese a ello, en el equilibrio entre los intereses del grupo y los intereses del individuo, la institución tendrá que ser siempre un anexo de los grupos de aprendizaje, y no su mentora, pues sólo así puede echar mano de su capacidad para obstruir los brotes de intolerancia y absolutismo apoyada en la moralidad colectiva de los grupos de aprendizaje.

El segundo fenómeno está vinculado con el funcionamiento de la moralidad colectiva. Por un lado, su forma de operación procura asentar todo aquello que logra un consenso ético dentro de la institución en fórmulas y diagramas administrativos y de planeación. Todo ello favorece la predominancia de una estructura para la supervisión y control constantes del individuo. El margen que se deja al criterio del individuo de aprendizaje es muy estrecho, y se relega la importancia de su sensibilidad y de su capacidad para resolver algún problema por su cuenta. El fin último de esta tendencia es el de poder anticiparse a cualquier eventualidad, posible o imposible, bajo una presencia aparentemente racional, sobria y tolerante.

Por otro lado, la evaluación inobjetable, sin posibilidad alguna de réplica o cuestionamiento, ya que lo contrario implicaría admitir intenciones desinhibidas del individuo, ha guiado invariablemente la práctica educativa participativa, y se ha caracterizado por representar algo así como una parte semiprivada que la institución preserva en compensación de la intolerancia hacia doctrinas dogmáticas o anticientíficas.

Uno de los problemas a los que se enfrenta la práctica educativa abierta de nuestros países es la carencia de una tradición democrática y de una vocación 
modernista. Y, como advertía Alfonso Reyes, lo único que puede generar tradiciones es la representación moral del mundo, a lo que agregaba, aceptando su exageración, es lo que nos ha faltado a los latinoamericanos. Más allá del desacuerdo que exista sobre el significado de la palabra moral, se debe enfatizar el sustrato de libertad que la sostiene y la proyecta: el acto docente abierto, así, puede ser entendido como la ejecución de una representación moral del mundo, en donde se congregan voluntariamente individuos que pretenden lograr objetivos de aprendizaje generalmente establecidos por una institución.

\section{EL PRINCIPIO DE LA LIBERTAD DE APRENDIZAJE COMO APORTACIÓN DE LA EDUCACIÓN ABIERTA}

América Latina fue en el siglo xx un espacio cultural en el que el sistema de educación superior estuvo consustanciado a las expectativas sociales, en el que la correspondencia entre lo que se desea ser y lo que se es estuvo marcada por la contradicción; por el festín de los proyectos y por el ayuno de la realidad.

Ni en Europa, ni mucho menos en los Estados Unidos, la orientación del sistema de la educación superior tuvo tantos referentes hacia la historia y la tradición, ni tampoco tanto impulso por transformarse y adquirir una dirección fiel a su naturaleza.

Sintetizadas en la Reforma Universitaria de Córdoba, desde la década de los veintes las demandas de transformación se expresaron a través de movimientos estudiantiles y magisteriales en diversos países como México, Chile, Cuba y Colombia.

Algunas de las principales demandas en esa década fueron:

Da autonomía de las universidades;

Da participación de los estudiantes en el gobierno de las universidades;

- La implantación de la docencia y la asistencia libres;

D La ampliación de la extensión universitaria, como medio de vinculación efectiva de las universidades con la vida social.

Cuando algunas de estas demandas se introdujeron a la vida cotidiana de las universidades, ya como prácticas institucionales como la ampliación de los cuerpos colegiados con la incorporación de algunos sectores de la comunidad que la integran, y los derechos del individuo ante la institución, como la libertad de cátedra, se empezó a hablar de la latinoamericanización de la educación, aunque más como un término despectivo que como uno laudatorio. Como refiere Ángel Rama, en no pocos congresos de educación europeos se proponían estrategias para evitar la latinoamericanización de sus instituciones educativas. 
Esta latinoamericanización refiere a un proceso más fácil de describir que de definir. Y es un proceso que aún está en marcha y que tuvo un nuevo impulso a fines de la década de los sesentas.

Cada una de las instituciones públicas de educación superior de Latinoamérica interpretó los signos de esta apertura en diversos niveles, y en su mayoría preservaron la idea de que la institución debía ser la tutora de los grupos de aprendizaje, induciendo sutilmente una vuelta hacia una práctica de comunicación inflexible, aunque participativa.

A diferencia de Estados Unidos, en Europa y en América Latina los movimientos estudiantiles estuvieron marcadamente politizados e inclinados hacia la izquierda, además de que se incluyó la autogestión como un punto político importante de la agenda de demandas hacia la institución y hacia el Estado.

El saldo positivo de esta oleada contra la organización del sistema de educación superior, fue que se modernizó el problema de la educación, es decir, que los grupos de aprendizaje cobraron presencia y adquirieron voz dentro de la institución. Pero en todo lo demás la sustancia de la organización de la educación no había desaparecido.

Uno de los proyectos que mejor reconoció este problema estructural del sistema de educación superior latinoamericano, y que propuso una alternativa viable, fue el del Sistema Universidad Abierta de la UNAM, creado en 1972, que de entre otros tenía como propósito transformar desde dentro la estructura de la educación escolarizada de la misma UNAM, de otras universidades públicas del país, y proyectar estas modificaciones al sistema de educación latinoamericano.

En sus fundamentos, trastocó la esencia de la organización de la educación conocida hasta entonces en América Latina: enfatizó el proceso del aprendizaje y procuró conciliarlo con los procedimientos de la enseñanza. Propuso reubicar los beneficios de la educación escolarizada, como los libros de texto y las cátedras, en conjunción con nuevas formas de transmisión, registro y evaluación de los conocimientos. Rescató principios como el de la libertad de cátedra y la evaluación colegiada, y apuntó otros como el que aquí denominaremos libertad de aprendizaje.

A diferencia de otras instituciones similares de la época, como la Open University inglesa, no tuvo que identificar ni proponerse ningún principio de apertura, pues la historia de su génesis ya estaba plena de ellos.

Si bien la libertad de cátedra ya había sido institucionalizada, en rigor este principio correspondería a la esfera de los procedimientos de la enseñanza.

El principio de la libertad de aprendizaje, en cambio, sólo fue señalado y limitado a la organización de los grupos de aprendizaje, pero su ejercicio sería del dominio únicamente del estudiante. 
Para que este principio pudiera tener plenitud de expresión, debía ajustarse a las condiciones impuestas para el ejercicio de la educación abierta, entre los más significativos:

D la opcionalidad del estudiante para seleccionar de entre múltiples recursos y condiciones dispuestos para propiciar el aprendizaje;

D el margen suficiente para disentir de los discursos institucionales, y

- las posibilidades de incluir nuevos miembros y nuevos recursos a los ambientes de aprendizaje.

Como se puede apreciar, este principio de la libertad del aprendizaje está vinculado con las posibilidades de infraestructura y de organización compleja que puedan ofrecer las instituciones educativas.

Asimismo, su desarrollo de grupos de aprendizaje a individuos de aprendizaje, sólo será posible por la incorporación de las tecnologías de la información y de la comunicación a los procesos educativos.

La organización hacia la que ya apuntan algunas instituciones de educación superior, como la UNAM, para consolidar este principio de libertad de aprendizaje, se está dando a través de proyectos de educación abierta y a distancia, y considera alguno o algunos de los siguientes aspectos:

- Aplicación de sistemas de selección y matriculación para una población heterogénea;

D Posibilidad de organizar los contenidos de los programas, a partir de contenidos propuestos por la institución;

D Alternativas para elegir métodos pedagógicos y recursos didácticos impresos, electrónicos o digitales;

Dacilidad para acceder al conocimiento en cualquier parte y en cualquier momento;

D Apoyo para seleccionar los métodos de evaluación del conocimiento;

Derecho a seleccionar de entre un sistema de asesores en las áreas:

- Disciplinaria,

- Psicopedagógica,

- Vinculación profesional

Dealimentaciones completas y frecuentes sobre el rendimiento académico;

D Inducción a la educación continua.

La libertad de aprendizaje es cada vez más una necesidad y cada vez menos un planteamiento, y para llevarlo a cabo hay que superar diversos problemas económicos, de organización y de administración institucional. 
Es probable que sólo las instituciones más inteligentes -en el sentido de recuperar la información necesaria para su propia transformación; las mejor preparadas para leer los datos de sus propias evaluaciones; las más dispuestas al cambio y las más ágiles, puedan enfrentar con éxito este tipo de demandas. Es probable también que no podrán hacerlo aisladas unas de otras.

La alianza interinstitucional es uno de los caminos que con mayor precisión se están consolidando en México, y que liderea la ANUIES a través de la formulación de su proyecto de creación de la Universidad Virtual mexicana, la que funcionaría con la suma de las fortalezas de las instituciones de educación superior, pero con personalidad propia y diferenciada de las partes.

Aunque el planteamiento de la estructura no es nueva, pues existen experiencias como el de la Open University finlandesa, que trabaja con esas características desde hace mucho tiempo, aquí uno de los principios que deben ser cuidados es precisamente el de la libertad de aprendizaje, el de la oportunidad de los estudiantes para manipular las estructuras del contenido curricular; para seleccionar de entre un grupo de asesores al que considere el más idóneo o capacitado; para seleccionar de entre un banco de recursos didácticos el que crea más adecuado y accesible a sus necesidades, etcétera.

En tanto que la educación abierta diseña patrones que pueden ser aplicados bajo diferentes condiciones, momentos, tecnologías y espacios, el principio de la libertad de aprendizaje permite redimensionar su posición frente a la sociedad y recuperar el sentido de innovación que paulatinamente ha abandonado a otras organizaciones.

La educación abierta funciona como tal únicamente cuando dota a cada individuo de un estatus y de una función, es decir, que lo ubique como miembro perteneciente a una comunidad, y que le facilite el desarrolle lo mejor de sus competencias o funciones. Un lugar y un rol, un ser y un estar. De su equilibrio depende el que haya una explicación del individuo desde la perspectiva del grupo, y una existencia del grupo desde la perspectiva del individuo.

La educación abierta es, ante todo, una estrategia para diseñar e implementar formas dinámicas de organización y de administración de la educación. El principio de la libertad de aprendizaje, así, tiene que observarse como un problema de organización institucional.

Sin embargo, la trayectoria que ha seguido la educación abierta está definida por el impulso inicial que la hizo posible: la de su desplazamiento como institución receptora a institución extensiva, e incluso como institución en desplazamiento, ya no en espera de que a ella acudan los estudiantes, sino de que ella acuda a los estudiantes, en donde quiera que estén, en el momento en que ellos lo definan, con las condiciones de que ellos dispongan, en los ritmos 
que ellos determinen. A esta respuesta es a la que he denominado libertad de aprendizaje.

La educación abierta, dentro del gran sistema educativo, debe tener clara su participación fundamentalmente para explotar las oportunidades, más que para resolver problemas. Del posicionamiento que pueda hacer al respecto dependerá el que rompa las ataduras que insisten en colocarlo sólo como un instrumento que ayude a recuperar la normalidad, es decir, sólo para resolver problemas. De aquí que los recursos y la organización deban estar dirigidos a las oportunidades, no a los problemas. Y la atención a la libertad de aprendizaje, en estos momentos, representa una gran oportunidad.

En suma, para que el principio de la libertad de aprendizaje pueda ejercerse es esencial que se produzca un intercambio y una combinación de información y de saberes. Con este fin, habría que promover y apoyar el diálogo y la colaboración entre docentes e investigadores, entre alumnos y entre escuelas y comunidades.

En segundo lugar, la utilización de las tecnologías de la información y la comunicación en la educación abierta debería proseguirse activamente, en particular para brindar oportunidades de aprendizaje permanente a nivel comunitario. A través de la diferenciación, la especificidad y el mejor control del alumno y el docente, estas tecnologías pueden satisfacer las necesidades particulares de la mayoría de los alumnos.

\section{LOS CAMINOS HACIA LA INDIVIDUALIZACION DE LA EDUCACIÓN}

La educación abierta funciona como tal únicamente cuando dota a cada individuo de un estatus y de una función, es decir, cuando lo ubica como miembro perteneciente a una comunidad, y que le facilite el desarrolle lo mejor de sus competencias o funciones. Un lugar y un rol, un ser y un estar. De su equilibrio depende el que haya una explicación del individuo desde la perspectiva del grupo, y una existencia del grupo desde la perspectiva del individuo.

La trayectoria de la educación abierta está definida por el impulso inicial que la hace posible: la de su desplazamiento como institución receptora a institución extensiva, e incluso como institución en desplazamiento, ya no en espera de que a ella acudan los alumnos, sino de que ella acuda a los alumnos, en donde quiera que estén, en el momento en que ellos lo definan, con las condiciones de que ellos dispongan, en los ritmos que ellos determinen.

Los problemas que debe resolver la educación abierta, por tanto, son exactamente los del abandono escolar, de ahí que cuando se observa que también en estos sistemas existe abandono, significa un doble fracaso, a menos que se admi- 
ta que existe un sector de personas que conservan la aspiración de continuar sus estudios, pero que aún bajo otras condiciones no son capaces de hacerlo, y que probablemente nunca lo hagan.

De esta manera, la educación abierta debe probar una sola cosa a la sociedad: su capacidad de acudir a cualquier lugar, en cualquier momento, en diferentes condiciones y ritmos, para ayudar a construir el conocimiento de quien lo requiera.

Si se quiere saber qué es la educación abierta, debemos comenzar con la identificación de su propósito, el cual se encuentra fuera de la misma educación abierta... en la sociedad, ya que las instituciones encargadas de desarrollar la educación abierta, son órganos de la sociedad. El propósito de la educación abierta, así, es... crear estudiantes permanentes.

El estudiante eventual esperaba vivir de lo que había conocido. El estudiante permanente espera una vida del conocimiento. Al estudiante eventual no le es ajeno el malestar del conocimiento, el estudiante permanente, en cambio, experimenta el malestar de la ignorancia. Cuando el malestar de la responsabilidad es mayor que el malestar de la ignorancia, el estudiante se separa de la institución.

Tal estudiante, sin embargo, al requerir trabajar apoyado en la asunción de su propia responsabilidad, le hace trabajar en contra de un ambiente pleno de distractores naturales, en contra de sus propias tendencias humanas dirigidas al ocio, que por otro lado deben ser recuperadas por la educación, que en su origen etimológico refiere precisamente al ocio. La mayoría de los estudiantes no lo hace, no sabe hacerlo o no puede hacerlo.

La condición del estudiante permanente comienza incluso antes de ser formalmente estudiante, cuando se recupera su experiencia, y continúa aún cuando ha abandonado la institución.

Si un alumno de educación abierta no acepta la responsabilidad, simplemente está condenado al fracaso. De aquí la importancia de la autoevaluación: el alumno tiene que comparar su desempeño con el estándar más alto posible: el suyo.

Si se quiere saber qué es la educación abierta, debemos comenzar con su propósito, el cual se encuentra fuera de la misma educación abierta: en la sociedad, ya que las instituciones encargadas de desarrollar la educación abierta, son órganos de la sociedad. Por ello el propósito de la educación abierta debe seres... crear estudiantes permanentes.

Tal estudiante, sin embargo, al requerir trabajar apoyado en la asunción de su propia responsabilidad, le hace trabajar en contra de un ambiente pleno de distractores naturales, en contra de sus propias tendencias humanas dirigidas al 
ocio, que por otro lado deben ser recuperadas por la educación, que en su origen etimológico refiere precisamente al ocio. La mayoría de los estudiantes no lo hace, no sabe hacerlo o no puede hacerlo.

Como la educación abierta ofrece servicios, es menester que invierta en el mejoramiento de ellos a través de la investigación. De donde los criterios para evaluar a la educación abierta deben estar siempre referidos al cumplimiento de los servicios, en lugar de la producción de egresados.

Ahora bien, ¿cómo puede participar, o mejor dicho, cómo participan los sistemas de educación superior abierta en el panorama del sistema educativo en general? Una de sus principales bondades es que tienen una naturaleza de dos caras: la flexibilidad y la anticipación; flexibilidad para adaptarse a los cambios, anticipación para preverlos.

Uno de los mayores cambios que se han dado en este sentido es el del conocimiento y el papel decisivo que ha alcanzado en la competencia mundial; estudios de prospectiva han indicado que en el 2020 tan sólo el volumen de la información se duplicará cada 73 días.

De allí que las tendencias señalen una demanda creciente de trabajadores altamente calificados que no sólo tengan conocimiento, sino que sepan cómo conseguirlo. En esas mismas tendencias se hace ver que el desempleo castigará en mayor medida a los que menos saben. Actualmente los graduados de las universidades tienen un porcentaje de desempleo que no es ni la mitad del que alcanzan quienes sólo estudian dos o tres años en la universidad. Los que no terminan la secundaria tienen un porcentaje de desempleo seis veces mayor que los graduados en las universidades.

Así, en términos generales, puede decirse que la fortaleza de los modelos de educación abierta, radica en su capacidad para adecuar las estructuras de organización académica y crear y revisar permanentemente sistemas de transmisión, evaluación y registro de conocimientos.

Históricamente, se ha demostrado que esta modalidad puede tener varios propósitos, de allí que uno de los primeros aspectos que deba resolverse es a quiénes se le prestarán los servicios. De aquí dependerá la forma de su estructura y de operación. De aquí dependerá también el diseño de las estrategias para garantizar su viabilidad y determinar los recursos y los métodos que se emplearán.

\section{UNA PROPUESTA FINAL}

Uno de los principales obstáculos para avanzar en la clarificación acerca de lo que debe hacer cada uno de los elementos que participan en la práctica edu- 
cativa abierta, y cómo hacerlo cada vez mejor, es el que se desprende precisamente de la confusión alrededor de los fines que ésta pretende alcanzar. De ahí que deba desplazarse la pregunta tradicional de la práctica educativa abierta, ¿qué es la educación abierta?, hacia una etapa más elemental, de forma que el de la práctica educativa abierta sea un problema centrado casi exclusivamente en aspectos técnicos, fundamentalmente en el intento de dar solución a la pregunta: ¿cómo debe organizarse la práctica educativa abierta para que sea posible la interpretación y la recreación del conocimiento?

La solución es entonces muy sencilla y aparentemente simple: se puede adquirir, transformar y extender el conocimiento con la participación integral de cada uno de los elementos constitutivos. La participación integral hace referencia sobre todo a la adición de la crítica y de la autocrítica como una fuente de regeneración de los vínculos que posibilitan la identificación de un elemento en otro. 


\section{REFERENCIAS BIBLIOGRÁFICAS}

Bertalanffy, L. V. (1975). Teoría general de los sistemas. México, FCE.

DANIEL, J. S. (1995) The Mega-universities and the Knowledge Media: implications of new technologies for large distance teaching universities. London, Open University.

DiRR, P.J. (1990). Distance education: policy considerations for the year 2000. En Moore, M. Ed. Contemporary issues in american distance education, Pergamon Press.

Escotet, M. A. (1980). Tendencias de la educación superior a distancia. Costa Rica, Editorial EUNED.

GonzÁlez Casanova, P. (1992). Los sistemas de universidad abierta y las ciencias y técnicas del conocimiento. En Herrero y Barrón, eds., Perspectivas del Sistema Universidad Abierta para el siglo XXI. Memorias. México, UNAM.

KeEgan, D. (1990). A theory for distance education. En Moore, M. Ed. Contemporary issues in american distance education, Pergamon Press.

Negroponte, N. (1995). Ser digital. Buenos Aires, Editorial Atlántida.

SEaRLE, J.R. (1985). Mentes, cerebros y ciencia. Madrid, Cátedra.

SEARLE, J.R. (1989). Las intenciones colectivas y las acciones colectivas. En Ibáñez Gracia, T. El conocimiento de la realidad social. Barcelona, Sendai ediciones.

SEARLE, J. R. (1995). ¿Crisis de la educación norteamericana?. En Vuelta, febrero, México.

SEARle, J. R. (2000). La misión de la Universidad: ¿descubrimiento intelectual o transformación social?. En Universum, año 15, Talca, Chile.

Villarroel, A. (1990). La educación a distancia: desarrollo y apertura. Caracas, ICDE - UNA.

\section{PERFIL ACADÉMICO Y PROFESIONAL DEL AUTOR}

Héctor Barrón Soto es Licenciado en Estudios Latinoamericanos. Jefe del Departamento de Organización y Seguimiento de Proyectos de la Dirección de Educación Abierta de la Universidad Nacional Autónoma de México. Ha publicado artículos especializados en educación abierta en revistas de la AIESAD y en The American Journal of Distance Education. Actualmente prepara un libro sobre la educación abierta en México. Fue Secretario Técnico del Programa de Transformación del Sistema Universidad Abierta de la UNAM en 1997.

Edificio Técnico de la Universidad Abierta de la UNAM, circuito exterior de Ciudad Universitaria, México, 04510. Teléfono 56228700 .

Correo electrónico hbarron@servidor.unam.mx 